\title{
Review of Various Aspects of Radio Frequency IDentification (RFID) Technology
}

\author{
Hussain Saleem ${ }^{1}$, M. Zamin Ali Khan ${ }^{2}$ and Shiraz Afzal ${ }^{3}$ \\ ${ }^{1}$ Department of Computer Science, University of Karachi, Karachi, Pakistan \\ ${ }^{2}$ Main Communication Network Department, University of Karachi, Karachi, Pakistan \\ ${ }^{3}$ Electronic Engineering Department, Sir Syed University of Engineering \& Technology, Karachi, Pakistan
}

\begin{abstract}
Radio Frequency Identification (RFID) is essentially a wireless method of identification of physically placed objects in a certain state space. It is the most emerging technology these days which is becoming the alternate for barcode. Hence this technology is receiving lot of attention comparatively over past few years. RFID technology is used to automatically identify any item or a package. Automatic identification can be done with the help of RFID Tags and RFID Reader units. An RFID tag contains the transponder that can transmit wireless information over short distance. This information is then received by the device called RFID Reader and further processed through middleware infrastructure. Billions of RFID tags are successfully installed worldwide. Tons of RFID Applications are being developed in the various fields like Healthcare, Engineering, Agri-food, Live-stocks etc. for traceability purpose. Familiarization with the benefit and the risk of RFID is important before using this technology. The shadow of this technology has made a huge impact on our daily lives. This text gives the brief review of RFID Technology with discussion on frequency ranges, standards, and applications with emerging innovations.
\end{abstract}

Keywords: RFID, Smart Tags, RFID Reader, Transponder, Antenna, Middleware, Traceability.

\section{INTRODUCTION}

The awaiting storms of potentially distracting technology have found ways to bring comfort in our lives by means of evolution of technology in various aspects. "Smart tags" or "Smart Chip" are interchangeable moniker for this technology. The technology is called Radio Frequency Identification (RFID) technology. The RFID tag is contained within or fixed on a product or a person for the detection, identification and traceability purpose using radio waves. RFID reader can read the information from several meters away. Privacy can be achieved by using this technology that marks an impact on our personal lives. The latent benefits of this technology are now one of our key concerns declaring the definite uses of RFID technology. Some of the features of this technology are illustrated in the following Figure-1.
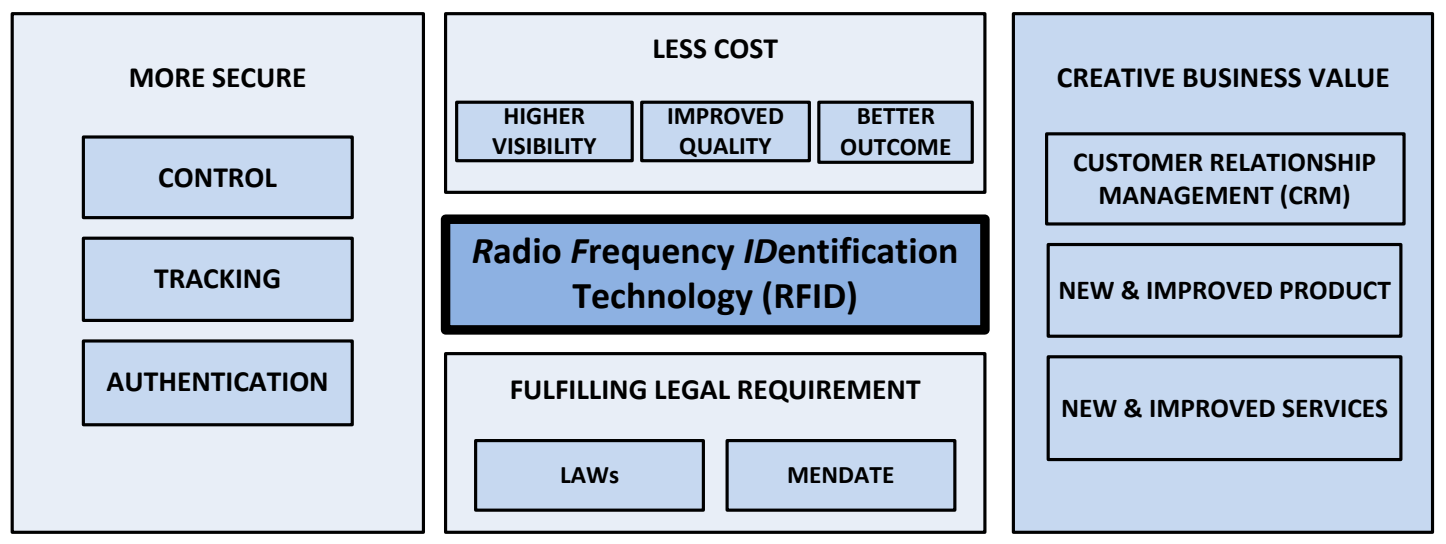

Figure-1: Illustration of some visible features of RFID Technology

With the specific Introduction in Section-I, Section-II covers the History of RFID. Section-III describes the basic infrastructure of RFID System. Section-IV explains the frequency-vise and Section-V gives construction-vise classification. In Section-VI, RFID Transponder Tag Code Structure and Standards are discussed. Section-VII describes the Security and Privacy issues regarding RFID technology. Section-VIII explains the various applications of this technology. The paper is concluded in Section-IX. 


\section{HISTORY OF RFID}

RF Identification technique has been used over number of years. The foundation of this technique was laid in 1940s and was deployed by the British Army to identify enemy aircraft [5]. Further in 1945 Leon Thermin invented surveillance tool for the Soviet Union that transmit the radio waves containing the audio information. The tool has a diaphragm, which is vibrated due to sound waves, which alters the shape of resonator. Although this device was not an identification tag but was a stealthy listening device, and therefore it was considered as a predecessor of RFID technology because this device was acting as a passive device that was energized by Electro-Magnetic waves (EM) generated by an external source [4].

Similarly in 1915, IFF transponder (Identification-of-Friend-or-Foe) was invented to identify the Aircraft in World War-II. Further in 1948, Harry Stockman explored the RFID technology in his paper titled as "Communication by Means of Reflected Power". In the late 1960s, the nuclear material safety and security issue causes the further development in RFID, as the invention of the compact disc which was envision in 1960s but didn't get enough popularity until 1980s.

In 1973, Mario Cardullo claimed that his passive radio transponder with memory was the first true precursor of today's RFID [3]. Further in 1973 the first demonstration of RFID tags were done at the Los Alamos Scientific Laboratory [2]. The major progress in RFID tracking was done in 1980s and 1990s. The Compaq computer started using RFID to track component through production assembly in 1980s.

Developments in the compactness of chips and enhancement in computer speed with complex topologies of computer network have broadly contributed to the increasing momentum of RFID. Most importantly, the standards are benchmarked during this development era to ensure that user can enjoy the ease of function and compatibility of this technology [14].

\section{RFID SYSTEM BUILDING BLOCKS}

The basic infrastructure of Radio Frequency Identification System comprises of two-way (Full Duplex) radio transmitter-receivers called Interrogator (Reader) and Transponder (Tag) as shown in the Figure-2. The RFID tag is composed of the transponder unit that contains the integrated circuit chip for processing and storing information, signal modulator/demodulator for modulating and demodulating radio frequency (RF) signal, and an antenna for transmitting and receiving signal. The information is further transferred to concerned workstations via transmission channel either wired or wireless network.

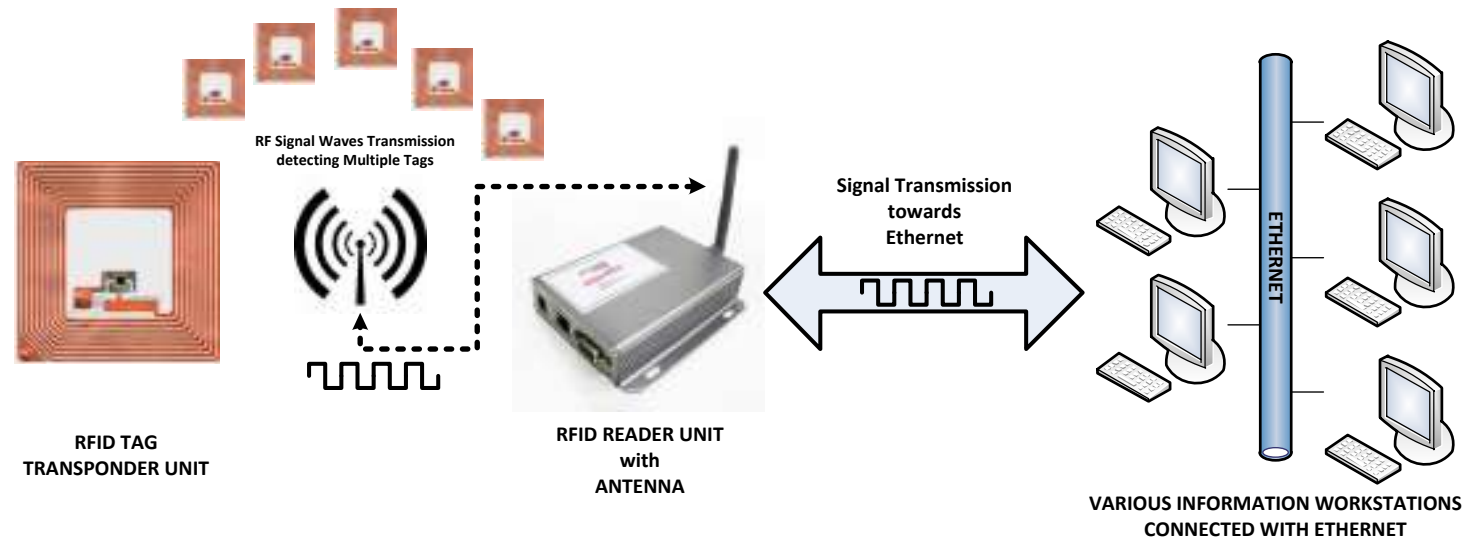

Figure-2: Basic RFID System Infrastructure

RFID tags are positioned on the assets to be tracked and monitored. The RFID tags transponder unit could be active or passive RFID depending on the asset type and its use. Active tags contain a battery, beacon to be charged on periodic basis. Depending on the type and power of antenna and noise in surrounding environment, the tags could be read remotely from distance of several hundred feet. In case of Passive tags, they derive their power while they are inside the signal range of the Reader. Since they are substantially less expensive than active RFID tags, they have noticeably less read range ability. Mostly having read ranges of 10 feet or less at maximum [8].

The RFID system is further connected with middleware software applications for business purposes like inventory or stock control, tracking humans in work-fields deployed on certain project in industry etc. The RFID events are processed by this middleware component and then presented to business application software where they are further processed. The RFID application software organizes the individual services in-line with process requirements when RFID reader is connected to host computer workstation via communication channel e.g. Ethernet as shown in Figure-2. The important thing is to ensure that all the tags having the same EPC 
(Electronic Product Code) are not allowed to read multiple times [11] and it also monitor the RFID hardware. One can easily communicate with the RFID tag using portable RFID reader like PDA or fixed RF reader which could be usually connected to complex computer network with the help of servers. The RFID tags which are successfully implemented worldwide are available in variety of style and shape, like a keychain, a credit card, a patient's wrist bracelet and dongle etc.

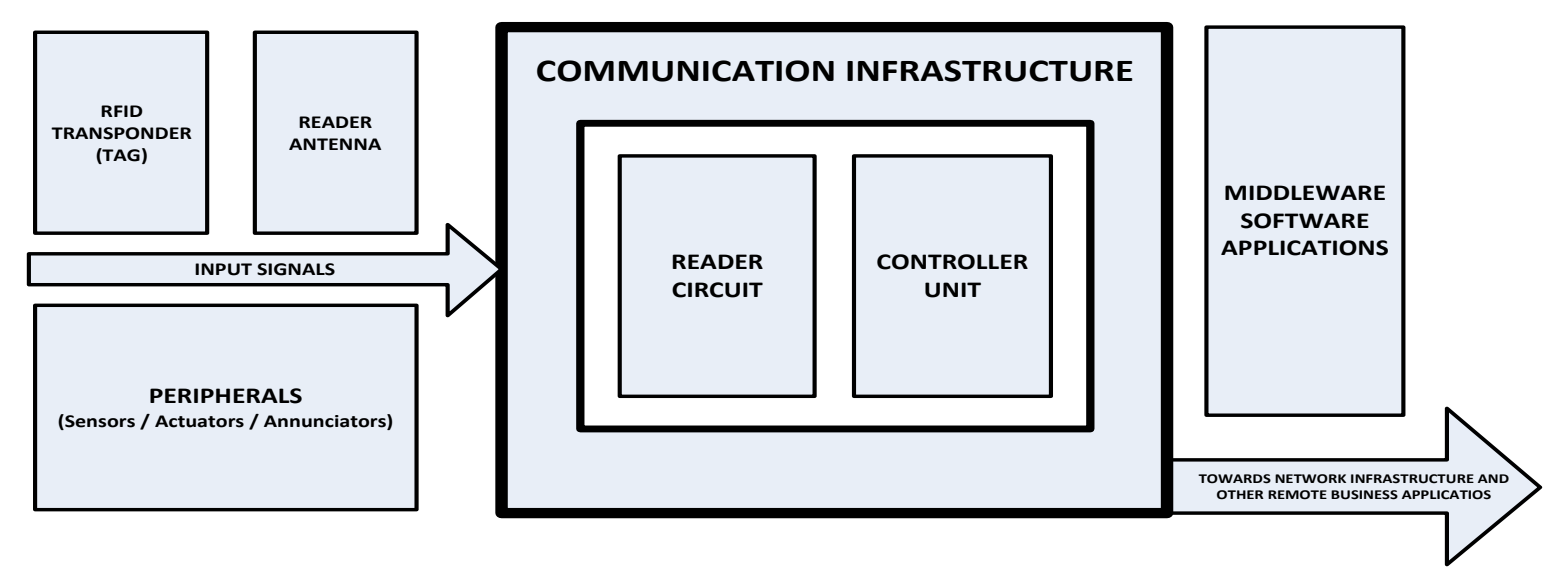

Figure-3: The Communication Infrastructure of RFID System with Inputs, Output as Middleware and other remote Business Applications connected to complex Computer Networks

The RFID System consists of the following components (in singular form) from an end-to-end perspective: (1) Tag or Transponder is a mandatory component of any RFID system. (2) Reader or Interrogator is also a mandatory component. (3) Reader Antenna, is another mandatory component. Some currently available Readers have built-in Antenna. (4) Controller Unit is also mandatory component. Most of the new generation readers have this component built-in to them. (5) Sensor, Actuator, and Annunciator. These optional components are needed for external input and output of the system. These are also called Peripherals. (6) Host Computer and Software System Workstations: Theoretically, an RFID system can function independently without this component. Practically, an RFID system is close to worthless without this component. (7) Communication Infrastructure: This mandatory component is a collection of both wired and wireless network and serial connection infrastructure needed to connect the external components together to effectively communicate with each other and compatible peripherals [6].

\section{FREQUENCY-VISE CLASSIFICATION FOR RFID}

The Frequency-vise classification of RFID i.e. frequency range slab is shown in Figure-5. Radio Frequency tags can either operate in the (1) Frequency range (100 kHz to $500 \mathrm{kHz})$ known as Low frequency RF tags or can operate in the (2) Frequency range from (10 MHz to $15 \mathrm{MHz}$ and $850 \mathrm{MHz}$ to $950 \mathrm{MHz}$ ) termed as High frequency RF tags. Further, it can also operate in the (3) Frequency range (2.4 GHz to $5.8 \mathrm{GHz})$ known as Ultra High frequency RF Tag. The Low frequency RF tags, which are economical and have slow speed as compared to High frequency RF tags, are usually used for the applications where the information transfer over short distance is required. In contrast, the High frequency RF tags can be used for medium range applications having faster speed and are used to transfer the information over long distance. The Ultra High frequency RF Tags are fastest then both Low frequency and High frequency RF tags but there is a requirement of line of sight for transmission.

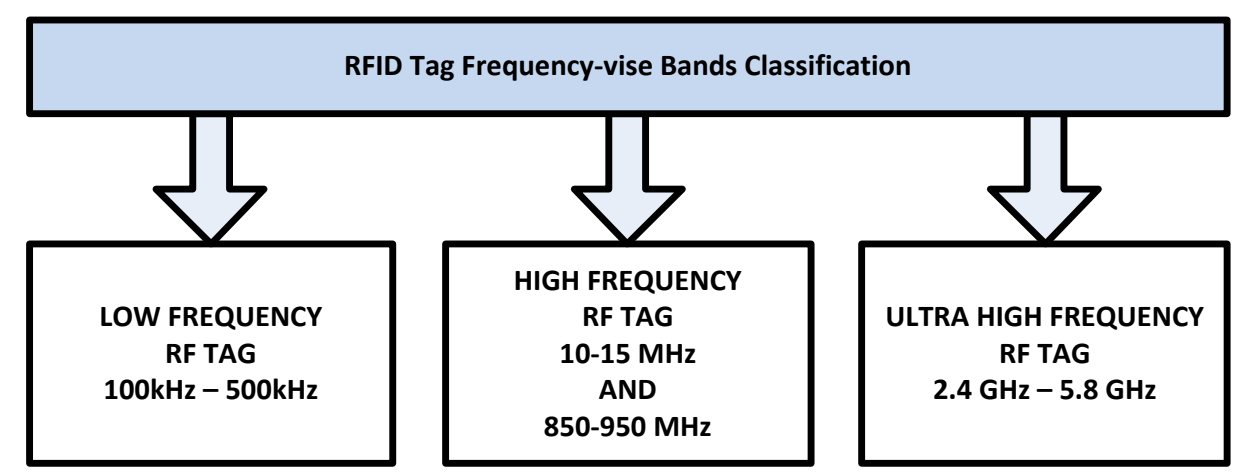

Figure-5: Block diagram showing the Frequency-vise Classification of RFID Technology 
Table-1 elaborate the relational parameters of frequency band, Regulations, Range of signal coverage in centimeters (cm) and meters (m), and comparative Data Speed of transmission [9][13].

\begin{tabular}{|c|c|c|c|}
\hline Band & Regulations & Range & Data Speed \\
\hline $120-150 \mathrm{kHz}(\mathrm{LF})$ & Unregulated & $10 \mathrm{~cm}$ & Low \\
\hline $13.56 \mathrm{MHz}(\mathrm{HF})$ & ISM Band Worldwide & $1 \mathrm{~m}$ & Low to Moderate \\
\hline $433 \mathrm{MHz}(\mathrm{UHF})$ & Short Range Devices & $1-100 \mathrm{~m}$ & Moderate \\
\hline $\begin{array}{c}868-870 \mathrm{MHz}(\text { Europe) } \\
902-928 \mathrm{MHz}(\mathrm{North} \text { America) UHF }\end{array}$ & ISM Band & $1-2 \mathrm{~m}$ & Moderate to High \\
\hline $2450-5800 \mathrm{MHz}$ (Microwave) & ISM Band & $1-2 \mathrm{~m}$ & High \\
\hline $3.1-10 \mathrm{GHz}$ (Microwave) & Ultra-wide Band & Upto $200 \mathrm{~m}$ & High \\
\hline
\end{tabular}

Table-1: Relational Parameters of RFID Frequency Band, Regulations, Range and Data Speed

\section{CONSTRUCTION-VISE Classification OF RFID TAGS}

The RFID tags are divided into two major categories. (1) Active RFID tags and (2) Passive RFID tags. Active RFID are the tags that include a battery as a power source and are true active since they are batteryassisted, while the Passive RFID tags are the one which have no internal battery power source as it is powered by Reader Unit and require higher power from Reader source. Passive RFID tags are smaller, lighter and less expensive; however Active RFID tags are comparatively expensive. Passive RFID tags have the long life i.e. almost unlimited life but short range coverage. While, in contrast Active RFID tags have short life due to the decay in battery life but have long range.

\section{RFID TRANSPONDER TAg CODE STRUCTURE AND STANDARDS}

The Electronic Product Code (EPC) is developed by EPC Global Consortium in order to track products through supply chain. EPC is one common type of data stored in a tag. It contains the basic structures of the Global Trade Item Number (GTIN) and Serialized Shipping Container Code (SSCC). The EPC when written into the tag by an RFID printer, the tag contains a 96-bit string of data. The first eight bits are a header which identifies the version of the protocol. The next 28 bits identify the organization that manages the data for this tag, the organization number is assigned by the EPC Global Consortium. The next 24 bits are an object class, identifying the kind of product; the last 36 bits are a unique serial number for a particular tag. These last two fields are set by the organization that has issued the tag. Similar as a URL, the total electronic product code number can be used as a key into a global database to uniquely identify a particular product [12].

\begin{tabular}{|c|c|c|}
\hline Frequency & Standard & Application \\
\hline $125 \mathrm{kHz}$ & ISO $18000-2$ & Used for detecting Animals \\
\hline $13.56 \mathrm{MHz}$ & ISO 14443 & Used to identify tangible objects like Book, Cloths etc. \\
\hline $400 \mathrm{MHz}$ & ISO $18000-7$ & Used for Vehicles Center Locking System \\
\hline $868 \mathrm{MHz}, 915 \mathrm{MHz} \& 922 \mathrm{MHz}$ & ISO $18000-6$ & Used for Inventory/Logistic Applications \\
\hline $2.45 \mathrm{GHz}$ & ISO $18000-4$ & Used for GPS Localization \\
\hline $5.8 \mathrm{GHz}$ & ISO $18000-5$ & Used for Vehicles identification and Highway Toll collection \\
\hline
\end{tabular}

Table-2: RFID Frequency Ranges, Standards, and their Key Applications [14]

A number of organizations have set standards for RFID, including the International Organization for Standardization (ISO), the International Electro-technical Commission (IEC), ASTM International, the DASH7 Alliance and EPC Global. There are also several specific industries that have set guidelines. These industries include the Financial Services Technology Consortium (FSTC) which has set a standard for tracking IT Assets with RFID, the Computer Technology Industry Association CompTIA which has set a standard for certifying RFID engineers, and the International Airlines Transport Association IATA which has set tagging guidelines for luggage in airports [14].

The ISO defines many RFID protocols at various frequencies. Various applications are using this protocol at different frequency ranges as shown in Table-2. The 18000 standards describe measurement procedures for checking RFID structures. Dash7 Alliance seeks to promote RFID Hardware based on ISO 18000-7 Standard [7]. 


\section{RFID SECURITY AND PRIVACY}

Security is a crucial aspect in RFID technology as the wireless communication has lot of security threats because in an open operation environment like RFID with widely distributed wireless access points could have more security risks as compared to normal computer wired network. Therefore RFID tags are inherently less secure due to the lack of processing capacity. Security threat on RFID is mainly considered in two different aspects such as (1) Any anonymous can disturb the functionality of the system remotely and (2) the other can affect the privacy of the user.

The RFID technology that is considered to be the wireless technology for identification could bump into different kinds of attacks or threats such as password hacking, sneaky scanning, cloning, functional creep and eavesdropping etc. as shown in Figure-6 as discussed in [1].

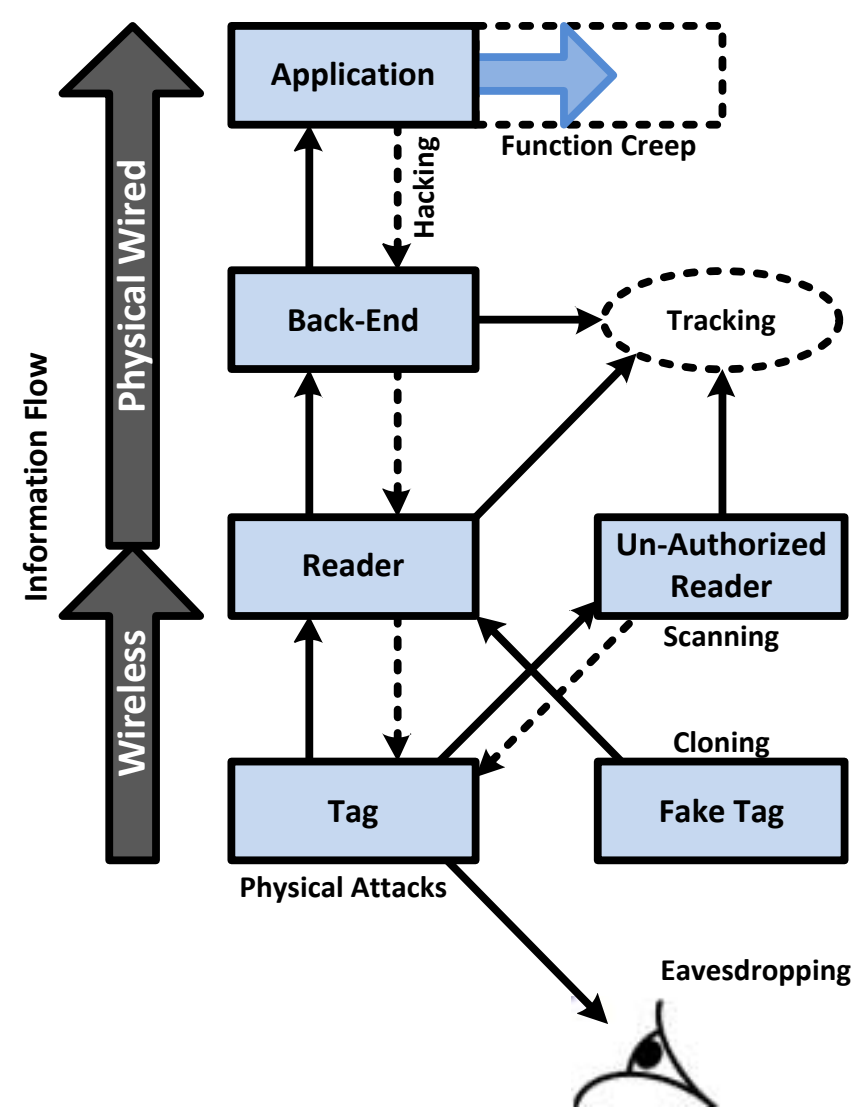

Figure-6: RFID Intrusion

\section{VARIOUS APPLICATIONS OF RFID}

RFID can be used in a variety of applications such as: (1) Access management, (2) Tracking of goods, (3) Tracking of persons and animals, (4) Toll collection and contact-less payment, (5) Machine readable travel documents, (6) Smart dust (for massively distributed sensor networks), (7) Location-based services, (8) Tracking Sports to verify authenticity, (9) Airport Baggage Tracking Logistics, (10) General Logistics, tracking a package or parcel, (11) Tracking vehicles for road pricing, (12) Passports development, (13) Medicine and drugs record, (14) Tags in clothing, e.g. in Jeans, shirts , coat and trouser etc., (15) Sealing for containers (for the shipping industry), and (16) Identifying animals, also used for tracking pets [10].

\section{CONCLUSION}

Remarkable potential is achieved using RFID technology up-till today not only making business welldisciplined, but also improved personal life standard of people. In the next few years more attractive applications will likely to be proliferating. Various aspects of RFID Technology have been discussed in this paper that clearly shows the value of this remarkable technology. 


\section{REFERENCES}

[1] A Soppera \& T Burbridge, "Wireless Identification - Privacy and Security”, BT Technology Journal, Vol.23, No.4, pp.54-64, (2005).

[2] Bitkom, “RFID: Technology, Systems and Applications", RFID Journal, Whitepaper, http://www.rfidjournal.com, (2005).

[3] Cardullo MW \& William LP, "Transponder Apparatus and System”, US 3713148, (Published May 21, 1970, Issued Jan 23, 1973).

[4] ISECOM, "Hacking Exposed Linux: Linux Security Secrets \& Solutions", (3/ed.), p.298, McGraw-Hill Osborne Media, (2008).

[5] Juels A., Molnar D., Wagner D., "Security and Privacy Issues in E-passports", SecureComm 2005, 1 st Intl. Conf. on Security \& Privacy for Emerging Areas in Communications Networks, pp.74-88, (2005).

[6] Lahiri S., "RFID System”, RFID Sourcebook, p.9, (2006).

[7] O'Connor, Mary Catherine, RFID Journal LLC, http://www.rfidjournal.com, (Published: March 18, 2009).

[8] Radiant Waves: Total RFID Solutions, Web: http://www.radiantwave.com/rfid_how.htm, (Accessed on: 30-09-2012).

[9] Sen D, Sen P \& Das AM, “RFID for Energy and Utility Industries", PennWell, pp.1-48, (2009).

[10] Southall M, Javick P, Lichtenberg J, \& Cusack BA, "Radio Frequency Identification: Transforming the Dynamics of Supply and Demand in Publishing", Publishing Research Quarterly, Vol.26, No.1, pp.1-10, (2010).

[11] TR3 Solutions, "Top 10 Myths about RFID Data Analytics", RFID Journal, http://www.rfidjournal.com, (2006).

[12] Vacca JR, “Computer and Information Security Handbook”, Morgan Kaufmann, p.208, (2009).

[13] Weis SA, "RFID (Radio Frequency Identification): Principles and Applications", MIT CSAIL, (2007).

[14] Wikipedia, “Radio-Frequency Identification”, Web: http://en.wikipedia.org, (Accessed: 30-09-2012).

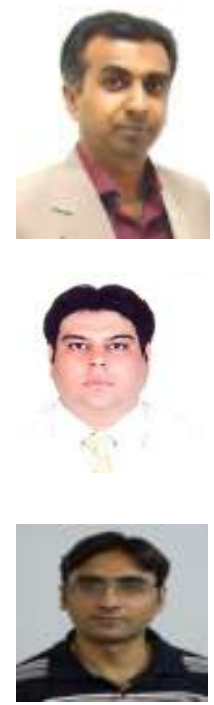

Hussain Saleem is currently Assistant Professor and Ph.D. Research Scholar at Department of Computer Science, University of Karachi, Pakistan. He received B.S. in Electronics Engineering from Sir Syed University of Engineering \& Technology, Karachi in 1997 and has done Masters in Computer Science from University of Karachi in 2001. He bears vast experience of about 16 years of University Teaching, Administration and Research in various dimensions of Computer Science. Hussain is the Author of several International Journal publications. His field of interest is Software Science, System Automation, Hardware design and engineering, and Simulation \& Modeling. He is senior member of Pakistan Engineering Council (PEC).

Dr. M. Zamin Ali Khan is a Head of Main Communication Network Department at University of Karachi. He has received B.E. (Electrical Engineering) from NED University, Karachi, Pakistan and M.S. (Electrical and Computer Engineering) from Concordia University, Montreal, Canada and Ph.D. in Computer Science from UoK. He has more than 18 years of experience of teaching and industry. He has worked in Victhom Human Bionics, Canada as an Engineer Scientist. Currently, he is a senior member of Pakistan Engineering Council, Canadian Engineering Council and IEEE. His research interest includes VLSI, Digital design, Digital signal processing and Analog front end of wireless devices.

Shiraz Afzal is a full time faculty member at Sir Syed University of Engineering \& Technology, Karachi, Pakistan. He received his B.S. in Electronics from Sir Syed University of Engineering and Technology Karachi, Pakistan and M.E degree in Electronics with specialization in Micro-System design from NED University of Engineering and Technology Karachi, Pakistan in 2006 and 2012 respectively. His research interest includes Microelectronic circuit design. He is also a member of PEC. 